\title{
Electrical characterization of PEDOT:PSS beyond humidity saturation
}

\author{
Mahmut Kuş ${ }^{\mathrm{a}, *}$, Salih Okur ${ }^{\mathrm{b}, * *}$ \\ a Selcuk University, Faculty of Engineering and Architecture, Department of Chemical Engineering, Kampus Selcuklu, Konya, Turkey \\ ${ }^{\mathrm{b}}$ Izmir Institute of Technology, Faculty of Science, Department of Physics, Urla, Izmir, Turkey
}

\section{A R T I C L E I N F O}

\section{Article history:}

Received 28 April 2009

Received in revised form 5 August 2009

Accepted 31 August 2009

Available online 8 September 2009

Keywords:

Humidity sensors

PEDOT:PSS

Resistivity

Conducting polymer

\begin{abstract}
A B S T R A C T
PEDOT:PSS humidity sensor was fabricated using a drop-casting method between thermally evaporated gold electrodes with $30 \mu \mathrm{m}$ separation and $300 \mu \mathrm{m}$ channel width on a glass substrate. AC, DC resistivity, and AFM techniques were used to characterize the PEDOT:PSS humidity sensor in the same environmental conditions. The change of resistivity was monitored with increasing relative humidity (RH) up to $90 \%$. The resistivity increases linearly up to a maximum value, and then it starts to decrease abruptly above $80 \%$ relative humidity $(\mathrm{RH})$ after saturation of water uptake. The decrease in resistivity above $80 \% \mathrm{RH}$ seems to be due to the water meniscus layer formed on the saturated PEDOT:PSS film. Below $80 \%$ RH, the device works like a humidity sensor.
\end{abstract}

(c) 2009 Elsevier B.V. All rights reserved.

\section{Introduction}

Conducting polymers (CPs) have been extensively used for various applications such as, light emitting diodes [1-2], photovoltaic cells [3], field-effect transistors (FETs) [4-6], and many types of sensors [7-12]. Due to their excellent physical and chemical properties such as relatively high electrical conductivity, ease of synthesis and design flexibility [1-2], CPs can be easily functionalized to improve the selectivity for different gases. Stability is one of the most important criteria for the application of organic [13-16] and inorganic devices [17-19]. Therefore, growths and stabilities of CPs have been extensively studied [13-16]. One of the well known, widely used conducting polymers is PEDOT:PSS. PEDOT itself is an insoluble polymer, but it becomes aqueous, if it is synthesized in the presence of poly(4-styrenesulfonate) (PSS). The polycationic PEDOT chains are incorporated into a polyanionic PSS matrix to compensate the charges. By doping PSS, PEDOT becomes hole conductive and it is used as a hole injection layer in organic electronics [1-3]. The change of conductivity depending on the environmental conditions such as humidity and temperature is very important for device operation. The influence of relative humidity $(\mathrm{RH})$ on electrical and optical properties of PEDOT:PSS has been studied extensively by several researchers [20-25]. Kawano et al. reported that the degradation of solar cell devices is a result of water absorption into the PEDOT:PSS layer [26]. This degradation of PEDOT:PSS layer appears

\footnotetext{
* Corresponding author. Tel.: +90 33222321 31; fax: +90 3322410635

** Corresponding author. Tel.: +90 23275077 06; fax: +90 2327507509.

E-mail addresses: mahmut_kus@yahoo.com (M. Kuş), salihokur@iyte.edu.tr (S. Okur).
}

to be spatially inhomogeneous and related with the formation of insulating patches causing loss of device current and consequently decrease in device efficiency. Huang et al. reported that exposure of the PEDOT:PSS layer to water results in an increase in the resistivity of the device, consistent with previous observations [27]. Polyamide fibers coated with PEDOT-PSS polymer material were also studied for humidity and temperature sensor applications [28]. Recently Liu et al. demonstrated the use of PEDOT-PSS humidity sensor application for detecting gravimetric water content in the soil samples [29]. They showed a linear increase in resistivity up to $80 \%$ RH value and then the resistivity of PEDOT-PSS humidity sensor with $50-\mathrm{nm}$ film thickness dramatically increased above that. However, we have obtained exactly different result above $80 \% \mathrm{RH}$. The increasing conductivity in our work might be originated from PSS content. Since PSS content in PEDOT is very important parameter for its conductivity. The thickness as a parameter is also very effective on transport properties of polymeric films.

In this study, we have reported the effect of relative humidity on DC and AC electrical resistivity of PEDOT:PSS film with 300$\mathrm{nm}$ thickness beyond the humidity saturation point around $80 \%$ for long lasting humidity sensor applications. For the first time, the interface mechanism for the sharply increasing conductance of PEDOT:PSS films after saturation point was investigated in detail.

\section{Experimental}

PEDOT:PSS (Baytron P 4083) was supplied from Bayer Co. Glass substrates were cleaned in acetone $30 \mathrm{~min}$ in ultrasonic bath before use. The thickness of PEDOT:PSS films were measured with a Dektak profilometer from Veeco and found to be $300 \mathrm{~nm}$. For the humidity 


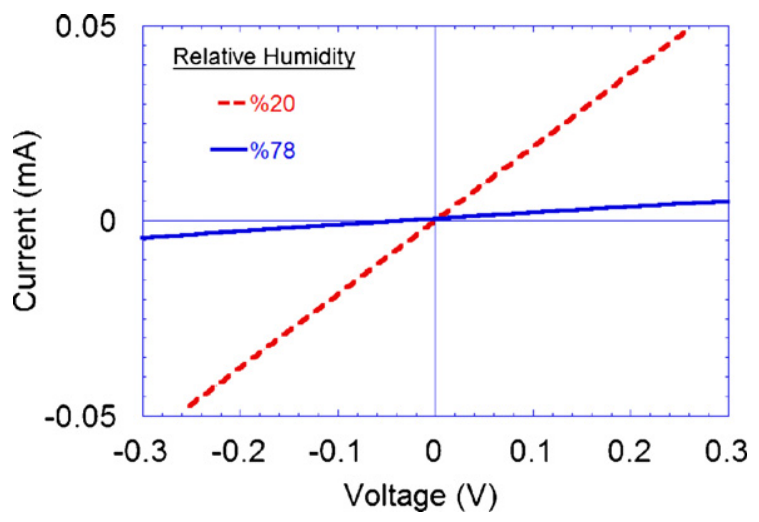

Fig. 1. $I-V$ characteristic of PEDOT:PSS with respect to relative humidity.

sensor measurements, gold metal electrodes (purity 99.95\%) with a thickness of $200 \mathrm{~nm}$ was thermally evaporated from a tungsten filament under $6 \times 10^{-6}$ Torr vacuum to form a channel length of $30 \mu \mathrm{m}$ and channel width of $300 \mu \mathrm{m}$ using a shadow mask.

A 10- $\mu$ I PEDOT:PSS suspension was drop casted between $\mathrm{Au}$ electrodes on a glass substrate and dried at room temperature. Electrical characterization was carried out with a Keithly 2420 source meter (Ohio, USA) at room temperature. The temperature change was measured around $24^{\circ} \mathrm{C}$ and kept constant. A homemade environmental chamber was used to control the humidity and temperature. A EI-1050 selectable digital relative humidity and temperature probe with a response time of $4 \mathrm{~s}$ and a resolution of $0.03 \% \mathrm{RH}$ was used with a USB controlled LabJack U12 ADC system combined with a single chip sensor module (SHT11) manufactured by Sensirion (Staefa, Switzerland). $200 \mathrm{mV}$ potential was applied and resistivity change was recorded with respect to various humidity levels. Resistivity measurements were also carried out with empty electrodes, water dropped empty electrodes and water dropped PEDOT:PSS coated electrodes.

The resistivity change was recorded for continuous humidity variations under 200-mV applied voltage. The morphology of the saturated and unsaturated PEDOT:PSS film on a glass substrate surface was examined with a Solver $\mathrm{P} 47 \mathrm{H}$ atomic force microscope (NT-MTD) (Moscow, Russia) operating in tapping mode in air at room temperature. Diamond-like carbon (DLC) coated NSG01 DLC silicon cantilevers (from NT-MTD) with a 2-nm tip apex curvature were used at the resonance frequency of $150 \mathrm{kHz}$. The Nova 914 software package was used to control the SPM system and for the analysis of the AFM images.

\section{Results and discussion}

The humidity response of PEDOT:PSS was tested with various parameters such as $I-V$ response, resistivity and relative humidity as a function of time and the resistivity during the desiccation and moistening process. Fig. 1 shows the $I-V$ characteristic of PEDOT:PSS at humidity level, $20 \%$ and $78 \%$, respectively. It is clear from Fig. 1 that the resistivity of PEDOT:PSS sharply increases from 5.3 to $65 \mathrm{k} \Omega$ by increasing humidity.

Fig. 2 shows the resistivity and relative humidity as a function of time. The resistivity increases in accord with $\mathrm{RH}$ up to $80 \%$ and then it starts to decrease when the moisture goes above it.

Crispin et al. reported that a single PSS chain interacts electrostatically over its length with many shorter PEDOT chains and the distance between adjacent PEDOT chains is small. Hence, this favors the hopping of charges between PEDOT chains. By increasing PSS ratio, the distance between PEDOT chains increases, so it leads to higher average hopping distance, and results in decrease of conductivity [30]. The resistivity of the PEDOT-PSS increases due to
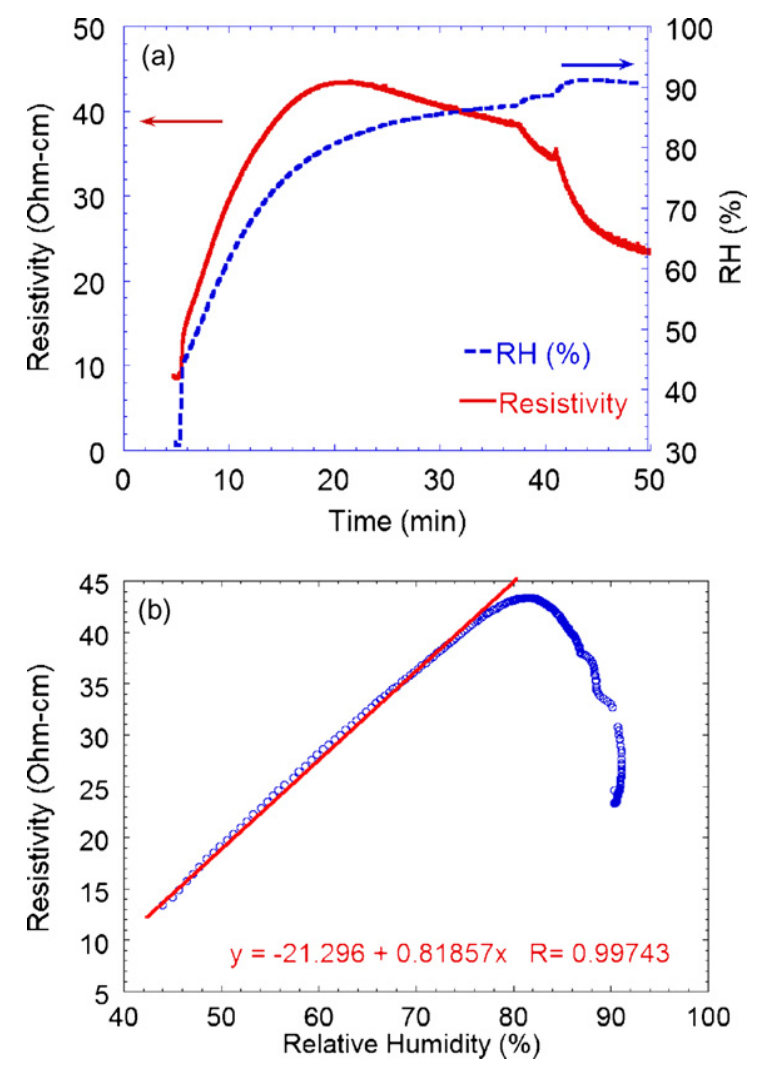

Fig. 2. Resistivity and relative humidity as a function of time (a) and resistivity versus relative humidity: the resistivity increases linearly up to $80 \% \mathrm{RH}$ and then decreases dramatically (b).

decreasing interaction between PEDOT chains as a result of swelling of the polymer by the water uptake under high relative humidity.

On the other hand, dipole moments of doping materials may also influence the conductivity [27]. Since the water molecule has a considerable dipole moment, the conductivity of PEDOT:PSS may decrease when water is absorbed by PEDOT:PSS [31].

The presence of water molecules causes an increase in distance between PEDOT chains and thus the electron hopping process becomes more difficult. Hence, the conductivity decreases. As mentioned above, an interesting point in our study is that, the resistivity increases linearly up to $80 \% \mathrm{RH}$ and then decreases dramatically above that value. This is most probably due to formation of water meniscus layer which dissolves PSS protons and also some atmospheric gases, such as $\mathrm{O}_{2}$ and $\mathrm{CO}_{2}$. Thus it leads to an increase in conductivity after the saturation point. By the formation of water meniscus layer at saturation point, the hole conductivity of PEDOT:PSS, which is effected by humidity, becomes ionic conductivity. Here, saturation point is referred to the humidity level around $80 \%$ at which resistivity reaches to a maximum value. We checked the formation of water meniscus with AFM The AFM images of PEDOT:PSS films before and after saturation of water uptake are given in Fig. 3.

As seen from the AFM surface topography given in Fig. 3(a) and (b), the small features of PEDOT:PSS film surface at $20 \%$ and $52 \% \mathrm{RH}$ are clearly visible and highly resolved, whereas the same features at $85 \% \mathrm{RH}$ are buried under the water meniscus layer and are barely visible in Fig. 3(c). It is clear that after saturation point, a water layer forms on PEDOT:PSS polymer surface and modifies the surface morphology. The surface morphology looks muddy due to water condensation above $80 \% \mathrm{RH}$. The rms surface roughness at $20 \% \mathrm{RH}$ was measured as $0.8 \mathrm{~nm}$ and increased to $1.2 \mathrm{~nm}$ at $85 \% \mathrm{RH}$. 

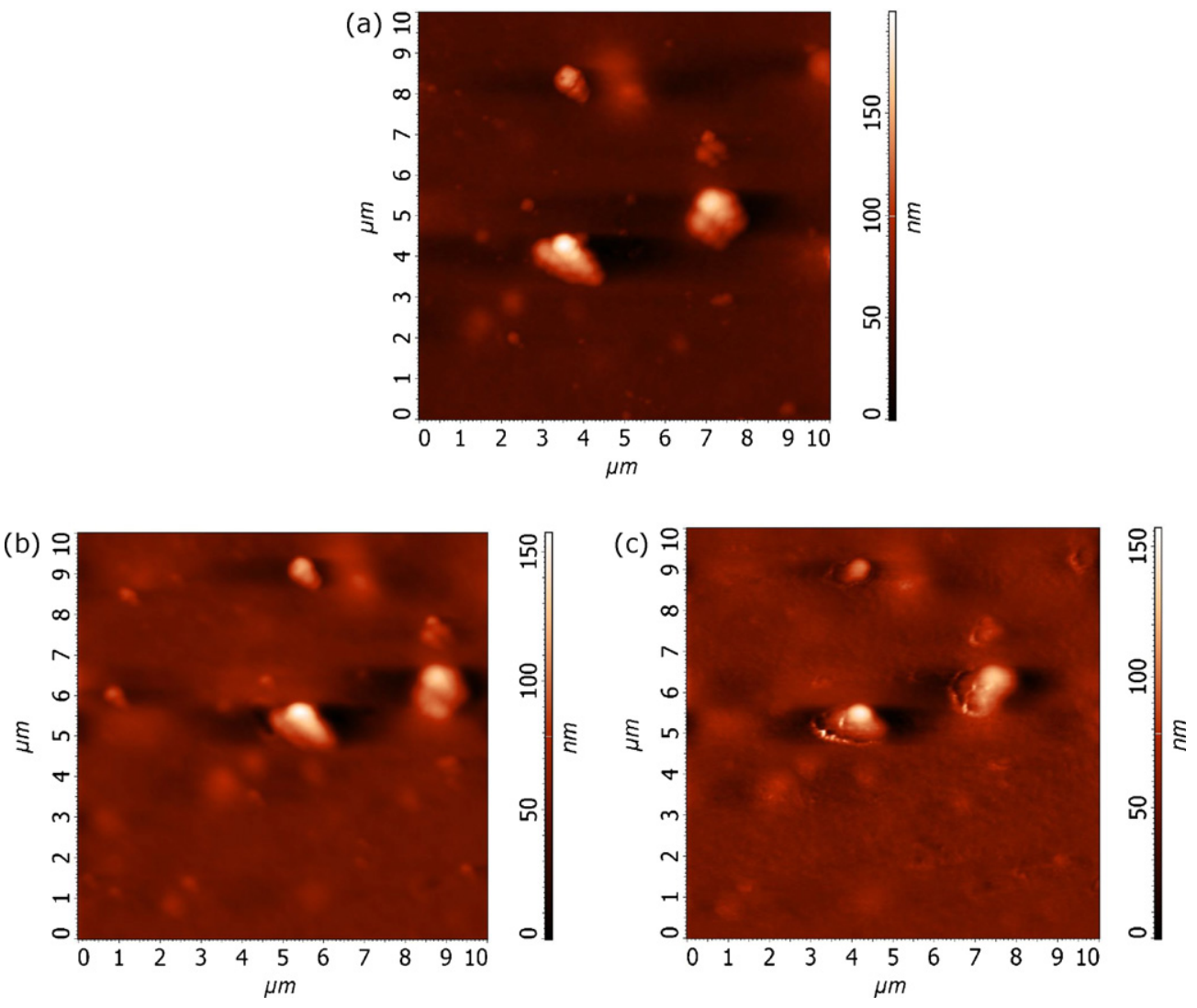

Fig. 3. AFM images of PEDOT:PSS films (a) at $20 \% \mathrm{RH}$, (b) at $52 \% \mathrm{RH}$, and (c) at $85 \% \mathrm{RH}$.

Although the formation of water meniscus up to $80 \% \mathrm{RH}$ was clearly seen with AFM, a water drop test has been also applied on an empty and a 300-nm thick PEDOT:PSS coated electrode with $30 \mu \mathrm{m}$ spacing, in order to see the effect of water condensation on the resistivity. Fig. 4(a) shows the water drop test on empty and PEDOT:PSS coated electrodes. The humidity response of an empty electrode with the same spacing has been also measured (Fig. 4(b)). From Fig. 4, it is clear that water drop shows a considerable decrease in resistivity at empty electrodes. This conductivity sources from the dissolving of some atmospheric gases such as $\mathrm{O}_{2}$ and $\mathrm{CO}_{2}$. Yatsuzuka et al. reported that the $\mathrm{O}_{2}$ and/or $\mathrm{CO}_{2}$ dissolve inside a water droplet and the conductivity of water droplet increases [32].

On the other hand, when a water droplet is put on PEDOT:PSS coated electrodes the resistivity decreases as expected. By comparing the water drop tests on empty and PEDOT:PSS coated electrodes, the resistivity of water dropped PEDOT:PSS coated electrodes shows 50 times smaller value than water dropped empty electrodes (see in Fig. 4(a)). It is of course due to the highly conductive PEDOT:PSS polymer but one must consider the conductance value of water droplet on electrodes. Dry PEDOT:PSS film shows about $65 \mathrm{ohm} \mathrm{cm}$ resistivity itself which is due to its own hole conductance but with water droplet this value decreases to $6 \mathrm{ohm} \mathrm{cm}$. As explained above, this decrease is most probably originating from both dissolving of PSS protons and also dissolving of atmospheric gases into the water layer which leads to an ionic conductivity. Hence, it should be expressed that, the ionic conductivity of water itself is negligible compare to the water droplet applied on PEDOT:PSS film.

It is also known that PEDOT:PSS suspension in water is highly conductive due to the acidic media $(\mathrm{pH} \sim 2)$ sourced from dissolving of PSS protons [33]. Beyond saturation point, the water layer dissolves PSS protons and thus the resistivity decreases quickly. To investigate the decrease in resistivity, all the devices were exposed
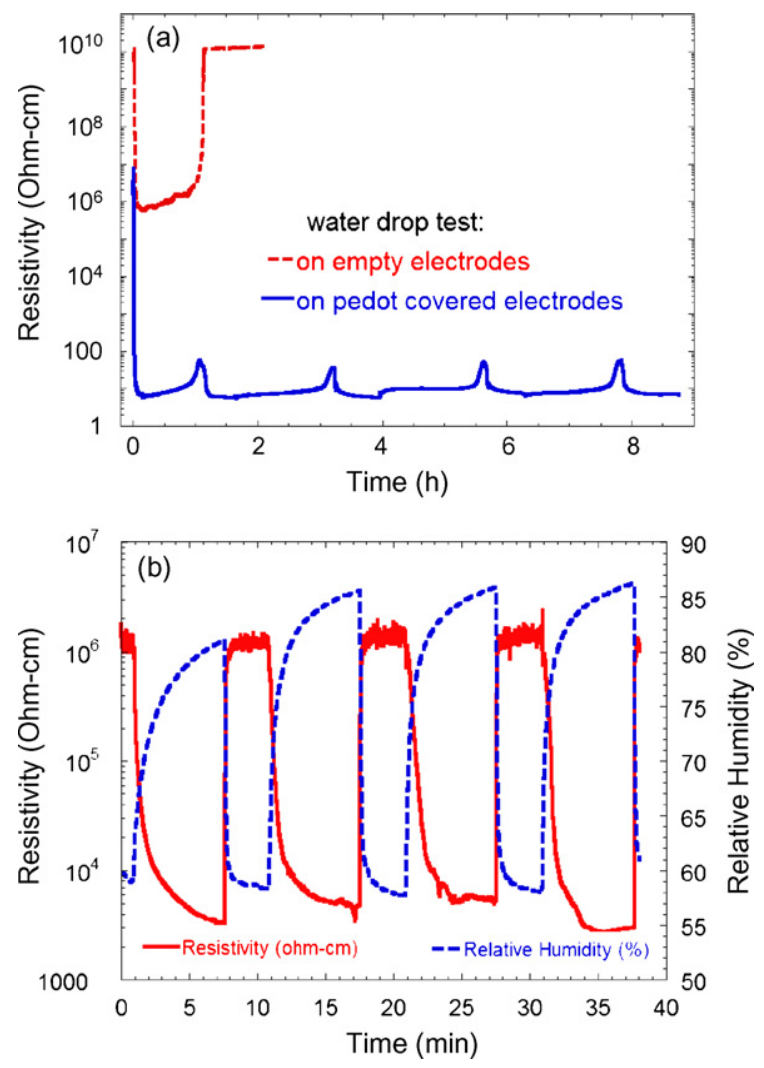

Fig. 4. Water drop test on an empty and a 300-nm thick PEDOT film covered electrode with $30 \mu \mathrm{m}$ spacing (a) and humidity response of an empty electrode with the same spacing (b). 


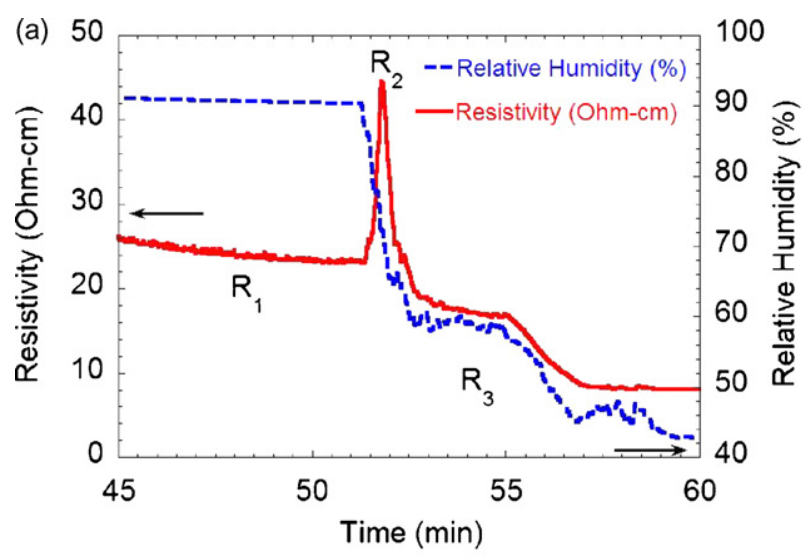

(b)
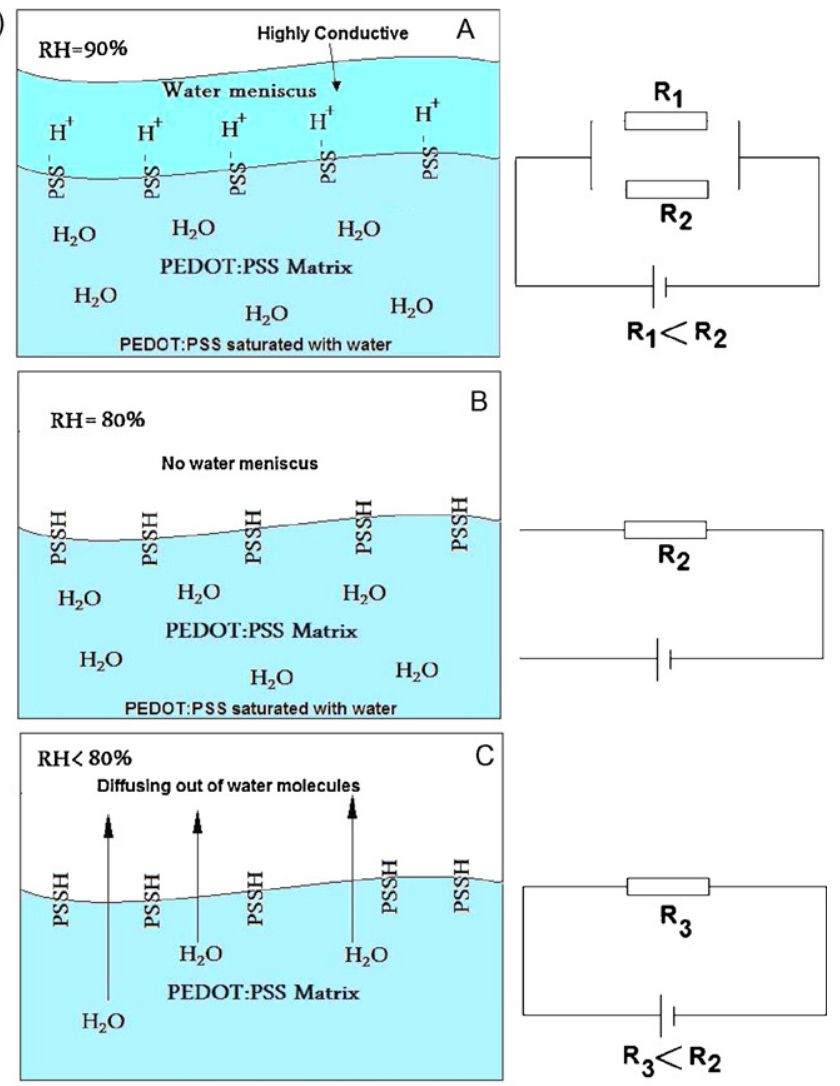

Fig. 5. DC resistivity and relative humidity (RH) as a function of time (a) and schematic explanation of the resistivity change of PEDOT:PSS film before and after water saturation point around $80 \%$ humidity (b).

to increasing moisture for several hours, and then checked their functionality. Fig. 5 shows the resistivity and humidity as a function of time. The device was exposed approximately an hour to the moisture and then desiccation process was applied. The resistivity looks nearly constant around $90 \% \mathrm{RH}$. When the desiccation starts, a fast increase and then decrease (a sharp peak) in resistivity is observed. Then the resistivity decreases in accord with decreasing $\mathrm{RH}$ below $80 \% \mathrm{RH}$. This sharp peak must be observed due to the humidity sensing character of PEDOT:PSS.

As mentioned above, a water layer forms on polymer surface and it causes the increase in conductivity and decreases the resistivity above $80 \% \mathrm{RH}$. The resistivity starts to increase quickly with decreasing $\mathrm{RH}$ due to the fast evaporation process of water meniscus layer from the PEDOT:PSS surface, which was acting like a resistor connected parallel between gold electrodes as shown in

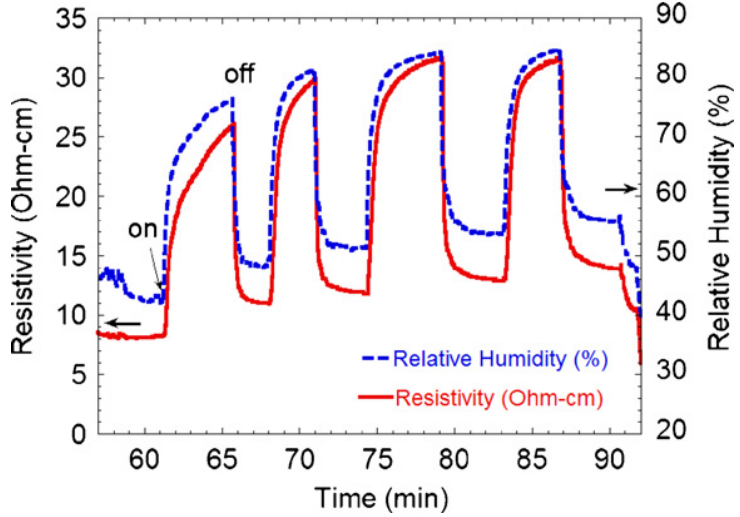

Fig. 6. The DC resistivity response time of the unsaturated PEDOT:PSS film to cyclic humidity change compared to the commercial humidity sensor.

Fig. 5((a) and (b)). Therefore, with a parallel resistor circuit analogy, the process can be explained easily as following: $R_{1}$ refers to the resistivity of the water meniscus layer and $R_{2}$ and $R_{3}$ refer to the resistivities of the saturated PEDOT:PSS layer and PEDOT:PSS layer below $80 \% \mathrm{RH}$, respectively. After evaporation of the water meniscus layer, the resistivity $R_{3}$ of the PEDOT:PSS film becomes related with the amount of water content inside the unsaturated PEDOT:PSS. Water molecules adsorbed by PEDOT:PSS starts to diffuse out of polymer matrix (Fig. 5(a) and (b)), so the resistivity $R_{3}$ starts to decrease. This means that $R_{2}$ is the maximum resistivity that the saturated or unsaturated PEDOT:PSS film can reach. Below $80 \% \mathrm{RH}$, the resistivity $R_{3}$ of the unsaturated PEDOT:PSS changes in accord with humidity level like a humidity sensor as shown in Fig. 6. The response time of PEDOT:PSS film to cyclic humidity change is relatively fast compared to the commercial humidity sensor as seen in Fig. 6. This result gives an idea about how a PEDOT:PSS device is stable and sensitive to the moisture as a humidity sensor below the saturation point (less than $80 \% \mathrm{RH}$ ).

The stability of device was also tested with DC and AC resistivity depending on desiccation and moistening process. It is clear from Fig. 6, reproducible humidity detection can be observed between $40 \%$ and $80 \% \mathrm{RH}$. The slowly increasing resistivity background level is due to the residual water content inside the polymer matrix during the process. This seems to be that the retention time at low humidity is not sufficient enough for the adsorbed water to be able to diffuse out of the PEDOT:PSS polymer matrix. However, the resistivity difference for each subsequent cycle is getting smaller with increasing on and off process time.

$\mathrm{AC}$ resistivity (impedance) change was also measured for similar cyclic humidity conditions between $40 \%$ and $90 \%$. Fig. 7 shows

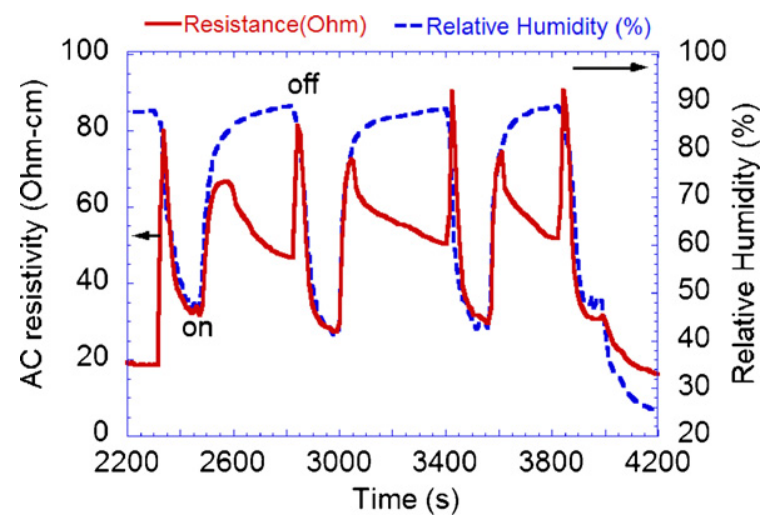

Fig. 7. The AC resistivity response time of the unsaturated PEDOT:PSS film to cyclic humidity change compared to the commercial humidity sensor. 
the AC resistivity and humidity as a function of time. Similar periodic fast response of the PEDOT:PSS film is also observed in the AC resistivity tests. The only difference between $A C$ and $D C$ resistivities is a sharp resistivity peak observed on each humidity cycle during desiccation and moistening process. The decrease in resistivity due to water meniscus layer above $80 \% \mathrm{RH}$ creates a resistivity peak, since the humidity cycle was chosen in the range between $40 \%$ and $90 \%$ to check the effect of water meniscus formation. On the other hand, there is no such periodic resistivity peaks in the DC measurements, since the humidity level was always below the saturation point $(80 \% \mathrm{RH})$. Considering the sharpness of the reverse peaks at the beginning of each desiccation process due to decrease of resistivity, the saturation of PEDOT:PSS film under AC voltage might be faster than under DC voltage, due to the vibration of polymer chains with the applied frequency of $40 \mathrm{~Hz}$, so that the water molecules can be easily diffuse in and out of the bulk PEDOT:PSS film.

\section{Conclusions}

The humidity saturation effect on the resistivity of a humidity sensor device based on PEDOT:PSS below and above $80 \%$ RH was analyzed in detail. A conductive water meniscus layer is formed on the saturated polymer films, so that it decreases the resistivity of PEDOT:PSS film due to the acidic feature of water meniscus. Our experimental results show that this water layer does not change the structural and electronic properties of the bulk PEDOT:PSS matrix permanently. The characteristic change in the resistivity below and above the saturation point is a reversible process. Hence, the PEDOT:PSS humidity device runs like a humidity sensor below $80 \%$ $\mathrm{RH}$.

\section{Acknowledgments}

This research is supported by TUBITAK (Scientific and Technical Research Council of Turkey) with the project number of TBAG108 T718 and by Turkish State Planning Organization (DPT) with the research project number of DPT2003K120390.

\section{References}

[1] J.H. Burroughes, D.D.C. Bradley, A.B. Holmes, Light-emitting diodes based on conjugated polymers, Nature 347 (1990) 539-541.

[2] H.S. Woo, S. Cho, T.W. Kwon, D.K. Park, Y.B. Kim, R. Czerw, D.L. Carroll, J.W. Park, Truly blue organic light-emitting diodes based on carbazole-doped 4,4'bis[carbazolyl-(9)]-stilbene, J. Korean Phys. Soc. 46 (2005) 981-984.

[3] G. Yu, J. Gao, J.C. Hummelen, F. Wudl, A.J. Heeger, Polymer photovoltaic cells: enhanced efficiencies via a network of internal donor-acceptor heterojunctions, Science 270 (1995) 1789-1791.

[4] A. Tsumura, H. Koezuka, T. Ando, Macromolecular electronic device: fieldeffect transistor with a polythiophene thin film, Appl. Phys. Lett. 49 (1986) 1210-1212.

[5] J. Lu, N.J. Pinto, A.G. MacDiarmid, Apparent dependence of conductivity of a conducting polymer on an electric field in a field effect transistor configuration, J. Appl. Phys. 92 (2002) 6033-6038.

[6] S.H. Jin, J.S. Yu, C.A. Lee, J.W. Kim, B.G. Park, J.D. Lee, Pentacene OTFTs with PVA gate insulators on a flexible substrate, J. Korean Phys. Soc. 44 (2004) 181-184.

[7] H. Zhang, S. Boussaad, N. Ly, N.J. Tao, Magnetic-field-assisted assembly of metal/polymer/metal junction sensors, Appl. Phys. Lett. 84 (2004) 133-135.

[8] G. Yu, J. Wang, J. McElvain, A.J. Heeger, Large-area, full-color image sensors made with semiconducting polymers, Adv. Mater. 10 (1998) 1431-1434.

[9] E.M. Genies, M. Marchesiello, Conducting polymers for biosensors, application to new glucose sensors GOD entrapped into polypyrrole, GOD adsorbed on poly(3-methylthiophene), Synth. Met. 57 (1993) 3677-3682.

[10] H. Sirringhaus, T. Kawase, R.H. Friend, T. Shimoda, M. Inbasekaran, W. Wu, E.P. Woo, High-resolution inkjet printing of all-polymer transistor circuits, Science 290 (2000) 2123-2126.

[11] D.T. McQuade, A.E. Pullen, T.M. Swager, Conjugated polymer-based chemical sensors, Chem. Rev. 100 (2000) 2537-2574.

[12] M. Gerard, A. Chaubey, B.D. Malhotra, Application of conducting polymers to biosensors, Biosens. Bioelectron. 17 (2002) 345-359.

[13] D.S. Park, Y.B. Shim, S.M. Park, Degradation kinetics of polypyrrole films, J. Electrochem. Soc. 140 (2002) 2749-2752.
[14] D.S. Park, Y.B. Shim, S.M. Park, Degradation of electrochemically prepared polypyrrole in aqueous sulfuric acid, J. Electrochem. Soc. 140 (1993) 609-614.

[15] Y.B. Shim, M.S. Won, S.M. Park, Electrochemistry of conductive polymers VIII, J. Electrochem. Soc. 137 (1990) 538-544.

[16] Y.B. Shim, S.M. Park, Electrochemistry of conductive polymers VII. Autocatalytic rate constant for polyaniline growth, Synth. Met. 29 (1989) 169-174.

[17] F. Finger, R. Carius, T. Dylla, S. Klein, S. Okur, M. Günes, Instability phenomena In microcrystalline silicon films, J. Optoelectron. Adv. Mater. 7 (2005) 83-91.

[18] F. Finger, R. Carius, T. Dylla, S. Klein, S. Okur, M. Günes, Stability of microcrystalline silicon for thin film solar cell applications, IEE Proc. Circ. Syst. Devices 150 (2003) 300.

[19] M. Egilmez, A. Gunel, S. Okur, M. Tanoglu, L. Ozyuzer, Electrical and microstructural properties of superconducting $\mathrm{MgB}_{2} / \mathrm{Mg}$ Composites, Key Eng. Mater. 264 (2004) 1197-1201.

[20] H.S. Kang, H.-S. Kang, J.K. Lee, J.W. Lee, J.Joo, J.M. Ko, M.S. Kim, J.Y. Lee, Humiditydependent characteristics of thin film poly(3,4-ethylenedioxythiophene) fieldeffect transistor, Synth. Met. 155 (2005) 176-179.

[21] Y. Kudoh, K. Akami, Y. Matsuda, Chemical polymerization of 3,4ethylenedioxythiophene using an aqueous medium containing an anionic surfactant, Synth. Met. 98 (1998) 65-70.

[22] Y. Kudoh, K. Akami, H. Kusayanagi, Y. Matsuda, Chemical polymerization of 3,4ethylenedioxythiophene in an aqueous medium containing a phenol derivative as an additive, Synth. Met. 123 (2001) 541-544.

[23] W.A. Daoud, J.H. Xin, Y.S. Szeto, Polyethylenedioxythiophene coatings for humidity, temperature and strain sensing polyamide fibers, Sens. Actuators B: Chem. 86 (2005) 329-333.

[24] D. Nilsson, T. Kugler, O. Svensson, M. Berggen, An all-organic sensor-transistor based on a novel electrochemical transducer concept printed electrochemical sensors on paper, Sens. Actuators B: Chem. 86 (2002) 193-197.

[25] A.M. Nardes, M. Kemerink, M.M. de Kok, E. Vinken, K. Maturova, R.A.J. Janssen, Conductivity, work function, and environmental stability of PEDOT:PSS thin films treated with sorbitol, Org. Electron. 9 (2008) 727-734.

[26] K. Kawano, R. Pacios, D. Poplavskyy, J. Nelson, D.D.C. Bradley, J.R. Durrant, Degradation of organic solar cells due to air exposure, Solar Energy Mater. Solar Cells 90 (2006) 3520-3530.

[27] J.S. Huang, P.F. Miller, J.S. Wilson, A.J. de Mello, J.C. de Mello, D.D.C. Bradley, Investigation of the effects of doping and post-deposition treatments on the conductivity, morphology, and work function of poly(3,4ethylenedioxythiophene)/poly(styrenesulfonate) films, Adv. Funct. Mater. 15 (2005) 290-296.

[28] W.A. Daoud, J.H. Xin, Y.S. Szeto, Polyethylenedioxythiophene coatings for humidity, temperature and strain sensing polyamide fibers, Sens. Actuators B: Chem. 109 (2005) 329-333.

[29] J. Liu, M. Agarwala, K. Varahramyan, E.S. Berney, W.D. Hodo, Polymer-based microsensor for soil moisture measurement, Sens. Actuators B 129 (2008) 599-604.

[30] X. Crispin, S. Marciniak, W. Osikowicz, G. Zotti, A.W. Denier Vander Gon, F. Louwet, M. Fahlman, L. Groenendaal, F. Deschryver, W.R. Salaneck, Conductivity, morphology, interfacial chemistry, and stability of poly(3,4-ethylene dioxythiophene)-poly(styrenesulfonate): a photoelectron spectroscopy study, J. Polym. Sci. B: Polym. Phys. 41 (2003) 2561-2583.

[31] P.M. Borsenberger, H. Bassler, Concerning the role of dipolar disorder on charge transport in molecularly doped polymers, J. Chem. Phys. 95 (1991) 5327-5331.

[32] K. Yatsuzuka, Y. Higashiyama, K. Asano, Electrification of polymer surface caused by sliding ultrapure water, IEEE Trans. on Industry Appl. 32 (1996) 825-831.

[33] J. Ouyang, C.-W. Chu, F.-C. Chen, Q. Xu, Y. Yang, Polymer optoelectronic devices with high-conductivity poly(3,4-ethylenedioxythiophene) anodes, J. Macromol. Sci. A: Pure Appl. Chem. 41 (2004) 1497-1511.

\section{Biographies}

Mahmut Kuş received his bachelor's degree in Chemistry from Selçuk University (Turkey) in 1998, his master's degree in Chemistry from Mugla University (Turkey) in 2001, and his PhD in Energy from Ege University Institute of Science in 2006. He is currently an assistant professor in Chemical Engineering at Selcuk University (Turkey). His current research interests include synthesis of organic and inorganic semiconductors and their applications in electronic devices such as organic photovoltaics, OLEDs, OTFTs and sensors.

Salih Okur received his bachelor's degree in Physics Education from Hacettepe University in Ankara, Turkey in 1989, his master's degree in Physics from Ankara University in Ankara, Turkey in 1992, and master's degree in Physics from Illinois Institute of Technology (IIT) in Chicago, IL, USA in 1996, and his PhD in Physics from IIT in 1998. He is currently an associate professor in the Department of Physics at Izmir Institute of Technology in Izmir, Turkey. His current research interests include SAM-LB-organic semiconducting thin film interfaces and their application to electronic devices such as organic photovoltaics, OLEDs, OTFTs, nano-dots, and nano-wires. 